\title{
Smart Portable Pen for Continuous Monitoring of Anaesthetics in Human Serum With Machine Learning
}

\author{
Simone Aiassa ${ }^{\circledR}$, Student Member, IEEE, Paolo Motto Ros ${ }^{\circledR}$, Member, IEEE, \\ Mandresy Ivan Ny Hanitra ${ }^{\circledR}$, Student Member, IEEE, Davide Tunzi, Maurizio Martina ${ }^{\circledR}$, Senior Member, IEEE, \\ Sandro Carrara ${ }^{\circledR}$, Fellow, IEEE, and Danilo Demarchi ${ }^{\circledR}$, Senior Member, IEEE
}

\begin{abstract}
Continuous monitoring of anaesthetics infusion is demanded by anaesthesiologists to help in defining personalized dose, hence reducing risks and side effects. We propose the first piece of technology tailored explicitly to close the loop between anaesthesiologist and patient with continuous drug monitoring. Direct detection of drugs is achieved with electrochemical techniques, and several options are present in literature to measure propofol (widely used anaesthetics). Still, the sensors proposed do not enable in-situ detection, they do not provide this information continuously, and they are based on bulky and costly lab equipment. In this paper, we present a novel smart pen-shaped electronic system for continuous monitoring of propofol in human serum. The system consists of a needle-shaped sensor, a quasi digital front-end, a smart machine learning data processing, in a single wireless batteryoperated embedded device featuring Bluetooth Low Energy (BLE) communication. The system has been tested and characterized in real, undiluted human serum, at $37^{\circ} \mathrm{C}$. The device features a limit of detection of $3.8 \mu \mathrm{M}$, meeting the requirement of the target application, with an electronics system 59\% smaller and $81 \%$ less power consuming w.r.t. the state-of-the-art, using a smart machine learning classification for data processing, which guarantees up to twenty continuous measure.
\end{abstract}

Index Terms-Anaesthesia, electrochemical sensor, embedded device, machine learning (ML), therapeutic drug monitoring (TDM).

Manuscript received December 11, 2020; revised February 17, 2021; accepted March 14, 2021. Date of publication March 19,2021; date of current version May 26, 2021. This work was supported by Politecnico di Torino and Compagnia di San Paolo under the initiative "Joint research Projects with top universities". This paper was recommended by Associate Editor Dr. Steffen Leonhardt. (Corresponding author: Simone Aiassa.)

Simone Aiassa is with the Department of Electronics and Telecommunications, Politecnico di Torino, 10129 Turin, Italy, and also with Integrated Systems Laboratory, École Polytechnique Fédérale de Lausanne, 1015 Lausanne, Switzerland (e-mail: simone.aiassa@polito.it).

Paolo Motto Ros, Davide Tunzi, Maurizio Martina, and Danilo Demarchi are with the Department of Electronics and Telecommunications, Politecnico di Torino, 10129 Turin, Italy (e-mail: paolo.mottoros@ polito.it; s243593@studenti.polito.it; maurizio.martina@polito.it; danilo. demarchi@polito.it).

Mandresy Ivan Ny Hanitra and Sandro Carrara are with the Integrated Systems Laboratory, École Polytechnique Fédérale de Lausanne, 1015 Lausanne, Switzerland (e-mail: ivan.nyhanitra@epfl.ch; sandro.carrara@epfl.ch).

Color versions of one or more figures in this article are available at https: //doi.org/10.1109/TBCAS.2021.3067388.

Digital Object Identifier 10.1109/TBCAS.2021.3067388

\section{INTRODUCTION}

$\mathbf{G}$ ENERAL anaesthesia is a medical procedure that requires the infusion of a perfectly balanced cocktail of drugs [1]. The procedure of anaesthesia presents numerous risks and side effects. As an example, the general sense of pain during anaesthesia has been reported frequently, in around $15 \%$ of patients [2], and it is usually related to under-dosage of anaesthetics. Meanwhile, other effects ranging from postoperative nausea to critical brain damages and death, are caused by over-dosage estimation of anaesthetics. The risk of death for anaesthesiology malpractices is estimated to be $0.001 \%$ [3]. Today, the anaesthesiology practice leverages on PharmacoKinetics and PharmacoDynamics (PK/PD) models with Target Controlled Infusion (TCI) pumps [4]. The patient is later monitored via BiSpectral (BiS)-index, a weighted sum of ElectroEncephaloGraphic (EEG) features [5]. This approach comes with some limitations since all TCI pumps implement PK/PD models experimentally derived from observation of drug effects on a population of individuals, being so only statistically accurate [6]. They cannot reproduce the interaction presents in the body of any individual. Closed-loop controlled infusion presents high advantages in general anaesthesia [7]. Several closed-loop devices are entering the clinical practice, but they are based on ECG [8], BIS index [9], or blood pressure sensors [10], with poor performances due to artefacts [11]. In the meantime, the loop between anaesthesiologists and patients may be closed with the Therapeutic Drug Monitoring (TDM). TDM provides help in determining the best cocktail of drugs, allowing a dynamic adjustment based on the response of the individual [12]. In this work, we present the piece of technology required to keep track of the infusion of anaesthetics, in real-time and with higher accuracy.

Propofol is the hypnotic agent usually administered to induce and maintain sedation in anaesthesia since it ensures fast and predictable time of effect [13]. A recent publication suggested that better control over propofol delivery is achieved with one measurement every half a minute, in the therapeutic range, i.e. between $10 \mu \mathrm{M}$ and $60 \mu \mathrm{M}$ of propofol, and with $10 \%$ accuracy around the target concentration [14]. Propofol concentration may be detected with non-invasive techniques in breath [15], [16], nevertheless, the determination of correlation between 


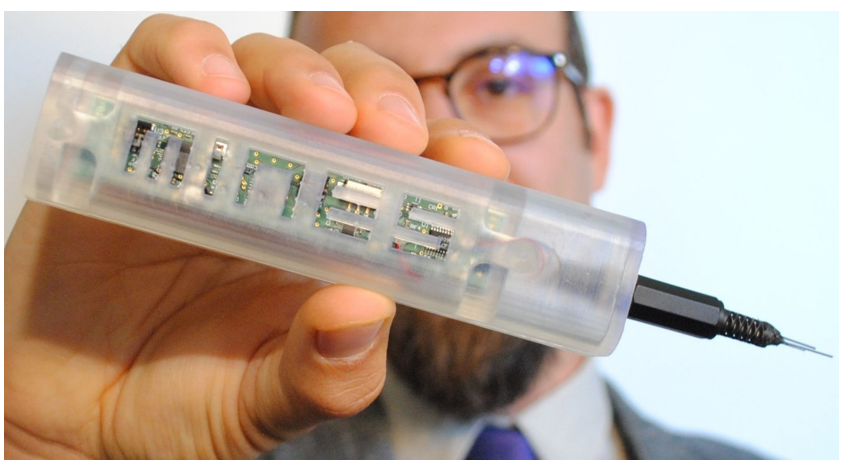

Fig. 1. The smart pen for direct and continuous classification of anaesthetics in human serum.

propofol concentrations in blood and breath presents another challenge [17], that is avoided with direct monitoring in human serum. For this reason, numerous electrochemical sensors have been proposed for detecting and measuring propofol in human serum or blood, achieving high sensing performance [18]. Unfortunately, they are disposable sensors for single-measure blood-spot sampling [19], and they are inefficient in an automatic TDM closed-loop system. Electrochemical determination of propofol suffers from the so-called fouling phenomena, which limits stability over time of the sensor. The electrochemical oxidation of propofol produces free radicals forming a polymeric film that covers the electrode and degrades the signal [20]. Solutions to cope with the propofol fouling of electrodes with specific materials are presented, without any sensor for in-situ detection [21], [22]. In addition to the sensor, a system allowing the monitoring of anaesthetics must include a potentiostat, i.e. the set of electronic components required to drive and read the electrochemical sensor [23]. Several circuits have been proposed for this purpose, but without considering long time detection of propofol [24], [25]. The systems proposed in literature for the monitoring of anaesthetics are usually big and power hungry [26] which limits their application in the surgery room, or they are designed as multi-purpose systems [27]. Moreover, smart data processing and display are generally missing.

In this work, we propose the first complete portable penshaped device for continuous real-time detection of propofol concentration in human serum to answer the request of such a device for monitoring anaesthetics toward safer anaesthesiology practices (See Fig. 1). The system has been fully characterized and tested for the classification of propofol concentration in the therapeutic range $(10 \mu \mathrm{M}: 60 \mu \mathrm{M})$ in human serum at $37^{\circ} \mathrm{C}$.

\section{THE SYSTEM}

The proposed complete system for anaesthetics monitoring is shown in Fig. 2. A needle-shaped electrochemical sensor, designed specifically for direct detection of propofol, is interfaced to the body of the patient under anaesthesia (on the right). The sensor connects to the smart portable pen (in the middle). The pen consists of a battery-operated embedded device packaged in a handy pen-shaped case. The device includes a Quasi Digital (QD) potentiostat as sensor front-end and communicates via
Bluetooth. On a computer (on the left), a Machine Learning (ML)-based model classifies the concentration of anaesthetics and informs the anaesthesiologist continuously on the concentration of propofol present in the body of the patient. The system input is the sample of human serum taken from the patient, while the output is the concentration of propofol displayed by the monitor. The electronic device and its case are fully novel. The QD potentiostat, the needle-shaped sensor, and the ML classifier were recently published and, for the first time, in this work, they are adapted to be inserted in a complete system.

\section{A. Needle-Shaped Electrochemical Sensor}

Propofol drug is an electro-active molecule, and it is detectable through electrochemical sensors. In particular, Cyclic Voltammetry (CV) techniques reliably determine its presence in human fluids [26]. In CV, a voltage staircase sweep is applied to an electrochemical cell across its Reference Electrode (RE) and its Working Electrode (WE). The potential triggers a Faradaic redox on the WE-interface, leading to a flow of electrons from WE to the Counter Electrode (CE). In the case of propofol, this current shows peaks, which are linearly proportional to the concentration of propofol, according to Randles-Ševčík equation [28].

The target TDM application requires a sensor which is disposable and that is used to continuously monitor the concentration of propofol in a flowing human fluid. The proposed sensor (Fig. 4) is a disposable low-cost three-electrode electrochemical cell in a needle shape to detect propofol in human serum presented in [29]. The electrochemical cell is composed of Pencil Graphite Electrodes (PGE), which reduces the propofol fouling of the surface [22]. The sensor sub-millimetre electrodes make the system less invasive and reduce the size of samples. The sensor $\mathrm{WE}$ is a $0.5 \mathrm{~mm}$ diameter $\mathrm{HB}$ mechanical pencil lead from Papeteria Migros, $10 \mathrm{~mm}$ long and active area around $15.9 \mathrm{~mm}^{2}$. The $\mathrm{CE}$ is another $0.5 \mathrm{~mm}$ diameter $\mathrm{HB}$ mechanical pencil lead, $15 \mathrm{~mm}$ long to guarantee a correct ratio between WE and CE area. The pseudo-RE is a $0.3 \mathrm{~mm}$ diameter, $8 \mathrm{~mm}$ long platinum wire. The sensor features a male audio jack $3.5 \mathrm{~mm}$ stereo connector, which provides a low cost, disposable, and robust electrical standard. The full assembly, the cleaning, and the activation of the electrode surface are detailed in [29].

\section{B. Quasi Digital Potentiostat}

The QD potentiostat leverages on the design presented in [30]. The circuit relies on analog to QD conversion, and vice-versa: the information is encoded in the temporal distance between consecutive pulses of a digital signal including both the properties of a digital and an analog signal [31], to maximize the quality-energy trade-off [32]. This event-based design avoids power-hungry Digital-to-Analog Converter (DAC) and Analogto-Digital Converter (ADC) [33], with a compact and low-power read-out circuit (even when a numerical output is needed [34]). Meanwhile, the event-based approach yields accuracy increase and noise reduction [35], and it provides advantages in the signal processing in the time domain; furthermore, the same 


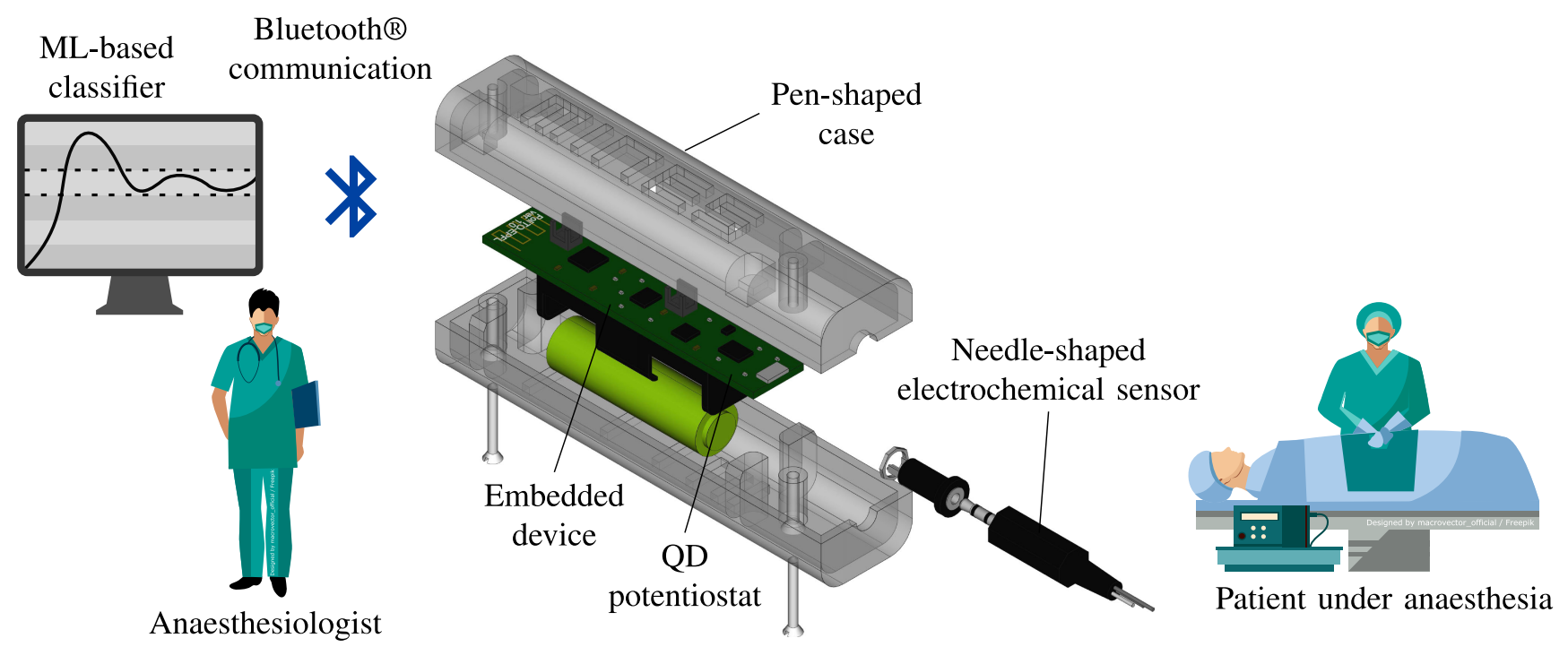

Fig. 2. The anesthesiologist follows up the infusion of anaesthetics in the patient body through the portable pen. The system includes a needle-shaped electrochemical sensor for propofol detection, a Quasi Digital (QD) potentiostat, on an embedded device, closed in a custom pen-shaped case, with Bluetooth; communication towards an external PC running a Machine Learning (ML)-based classifier.

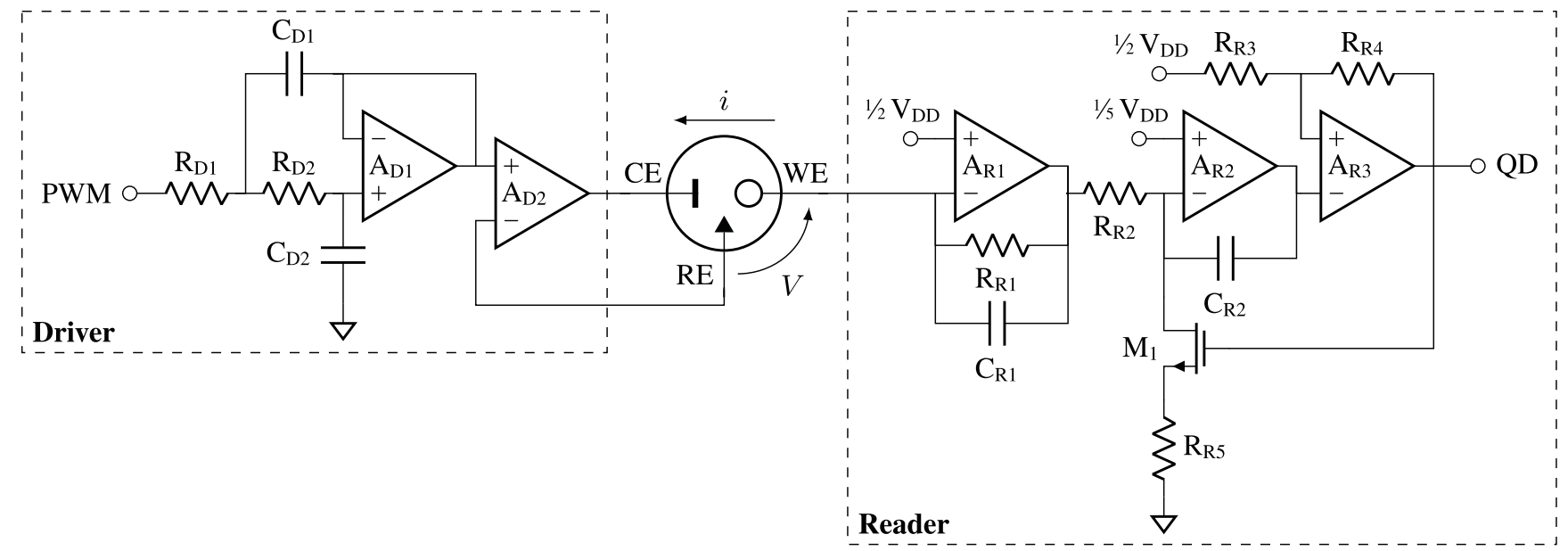

Fig. 3. Circuit implementation of the QD potentiostat. The driver (on the left) converts the Pulse Width Modulated (PWM) input in the potential to be applied cross RE and WE, while the reader (on the right) converts the Faradaic current flowing through WE and CE into the QD output.

approach can be seamlessly applied to the (wireless) information transmission as well [36].

Fig. 3 displays the circuit of the proposed QD potentiostat. The electrochemical cell is in grounded WE topology to minimize the number of components required [30], with a voltage driver and a current reader. The virtual ground of the electrochemical cell (WE) is connected to half supply voltage to avoid the introduction in the system of a dual-supply. The circuit is composed of a potentiostat driver to drive the CV staircase upon RE and CE electrodes of the electrochemical cell and trigger the Faradaic reaction. Meanwhile, the potentiostat reader inputs the Faradaic current flowing between WE and CE and which is the target of the measurement system.

In the potentiostat driver, the potential across RE and WE of the electrochemical cell is driven by a $20 \mathrm{kHz}$ Pulse Width Modulated (PWM) digital signal, consisting of a series of digital pulses modulated in its width. The PWM is filtered into a continuous voltage by a second-order active low-pass filter in the Sallen-Key configuration $\left(\mathrm{A}_{\mathrm{D} 1}\right)$ at the cut-off frequency of $33 \mathrm{~Hz}$ and quality factor around 0.8 . The filter output voltage is directly proportional to the Duty Cycle $(D)$ of the PWM signal according to Eq. 1, and the CV driving potential sweep is obtained by modulating $D$. The voltage follower $\left(\mathrm{A}_{\mathrm{D} 2}\right)$ avoids current flow to the RE, standard practice in grounded-WE configuration to avoid resistive leakage current.

$$
V=D \mathrm{~V}_{\mathrm{DD}}+V_{0}
$$

The detection of anaesthetics is obtained by analyzing the Faradaic redox current $(i)$ flowing from the WE to the CE. In the potentiostat reader, the trans-impedance amplifier $\left(\mathrm{A}_{\mathrm{R} 1}\right)$ adapts the $i$ current to the dynamic. The feedback capacitor $\left(\mathrm{C}_{\mathrm{R} 2}\right)$ is discharged according to the input current through the integrator 


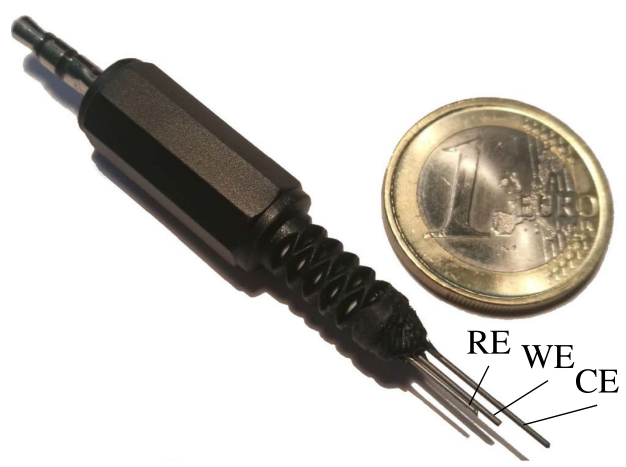

Fig. 4. Disposable pencil graphite needle-shaped sensor for detection of propofol in human serum.

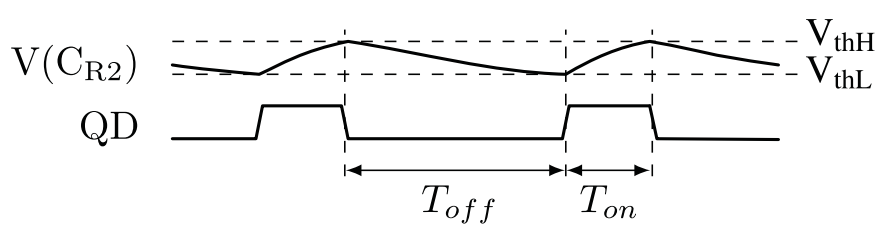

Fig. 5. Waveform of the current-to-QD conversion: the capacitor $\mathrm{C}_{\mathrm{R} 2}$ is discharged according to the input current, after a time $T_{o f f}$, the comparator sets the $\mathrm{QD}$ signal when $\mathrm{V}\left(\mathrm{C}_{\mathrm{R} 2}\right)$ overcomes the lower threshold $\left(\mathrm{V}_{\mathrm{thL}}\right)$. After a time $T_{o n}, \mathrm{C}_{\mathrm{R} 2}$ is charged again to $\mathrm{V}_{\mathrm{thH}}$ and $\mathrm{QD}$ is reset.

$\left(A_{R 2}\right)$. As shown in the waveform in Fig. 5, the comparator with hysteresis $\left(\mathrm{A}_{\mathrm{R} 3}\right)$ sets the $\mathrm{QD}$ output signal when the voltage across $\mathrm{C}_{\mathrm{R} 2}$ overcomes the lower threshold $\left(\mathrm{V}_{\mathrm{thL}}\right)$, after a $T_{\text {off }}$ time. When QD is high, a reference current flowing through the feedback MOSFET $\left(\mathrm{M}_{1}\right)$ and $\mathrm{R}_{\mathrm{R} 5}$ quickly load $\mathrm{C}_{\mathrm{R} 2}$. After a $T_{\text {on }}$ time, $C_{R 2}$ is loaded over the higher threshold $\left(V_{t h H}\right)$ imposed by $\mathrm{A}_{\mathrm{R} 3}$, the signal QD goes low, and the conversion starts again. The target current $(i)$ is proportional to the temporal distance (time $T_{o f f}$ in seconds) between a falling edge and a rising edge of the QD signal according to Eq. 2, where $i_{0}$ is the offset current.

$$
i=-\frac{\mathrm{R}_{\mathrm{R} 2} \mathrm{C}_{\mathrm{R} 2}\left(\mathrm{~V}_{\mathrm{thH}}-\mathrm{V}_{\mathrm{thL}}\right)}{\mathrm{R}_{\mathrm{R} 1} T_{\text {off }}}+i_{0}
$$

$A_{D 1}, A_{R 1}, A_{R 2}, A_{R 3}$ are implemented with the integrated op-amp Analog Devices AD8630, which features low offset and drift, with high bandwidth. $\mathrm{A}_{\mathrm{D} 2}$ is implemented with LTC; 6085 , which guarantees $1 \mathrm{pA}$ of input bias current to reduce $\mathrm{RE}$ parasitic current. $\mathrm{R}_{\mathrm{R} 3}$ is equal to $\mathrm{R}_{\mathrm{R} 4}, \mathrm{~V}_{\mathrm{thL}}$ is $1 / 4 \mathrm{~V}_{\mathrm{DD}}$ and $\mathrm{V}_{\mathrm{thH}}$ is $3 / 4 \mathrm{~V}_{\mathrm{DD}}$. $T_{\text {on }}$ is $2 \mu \mathrm{s}$ (according to Eq. 3) in order to be lower than $T_{\text {off }}$ while guarantying a glitch-free electronics. The input current maximum range is between $-60 \mu \mathrm{A}$ and $60 \mu \mathrm{A}$. In this range, $T_{\text {off }}$ spans between $29.4 \mu$ s and $2.63 \mathrm{~ms}, 58.8 \mu$ s at zero input current.

$$
T_{\text {on }}=2 \mathrm{R}_{\mathrm{R} 5} \mathrm{C}_{\mathrm{R} 2}
$$

\section{Embedded Device}

The core of our portable smart pen is a custom-built embedded device enclosed in a 3D-printed pen-shaped case. The embedded device consists of a single, double-layer Printed Circuit Board $(\mathrm{PCB})$ of size $92 \times 17 \mathrm{~mm}$. The case is a box-shaped container

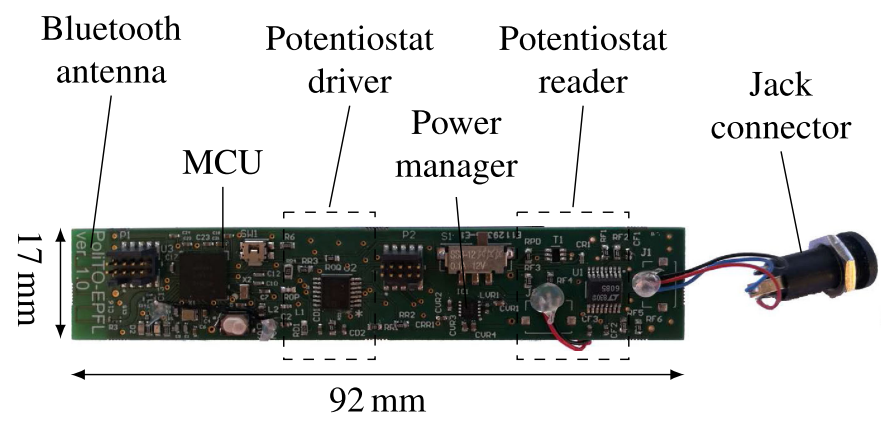

Fig. 6. The embedded board includes on a single PCB a Bluetooth; antenna for communication, a MCU for processing, the QD potentiostat (driver and reader), a power manager, and the jack connector to host the sensor.

with rounded corners of size $130 \times 30 \times 30 \mathrm{~mm}$. The PCB (Fig. 6) includes a Bluetooth; antenna for wireless communication, a MicroController Unit (MCU) for smart detection, the previously mentioned QD potentiostat (driver and reader), and a power manager. The PCB is connected to the disposable sensor with a jack connector.

The MCU is the Nordic Semiconductor nRF52840 built around the $32 \mathrm{~b}$ ARM; Cortex ${ }^{\mathrm{TM}}-\mathrm{M} 4$ processor, with a floatingpoint unit at $64 \mathrm{MHz}$. This component provides sufficient processing power to guarantee future development in a fully embedded framework, and it already showed excellent capability in biomedical applications [37]. The QD potentiostat is implemented on the top of the PCB with Commercial-Off-The-Shelf (COTS) components, to reduce cost and increase flexibility. The potentiostat is interfaced on one side to the electrochemical needle-shaped sensor through the female audio jack connector. On the other side, PWM and QD signals are connected to two MCU General Purpose I/O (GPIO) ports. The system features Bluetooth 5, IEEE 802.15.4-2006, 2.4 GHz transceiver to allow Bluetooth Low Energy (BLE) communication. The Bluetooth antenna is integrated on the PCB top layer as a copper trace antenna. A lithium-thionyl chloride AA $3.6 \mathrm{~V}$ battery with a capacity of $2700 \mathrm{mAh}$, placed conveniently on the backside of the PCB, powers the device. A buck-boost DC-DC voltage regulator, Texas Instrument ${ }^{\mathrm{TM}}$ TPS63031, fixes the output voltage at $3.3 \mathrm{~V}$ [38].

The MCU hosts a firmware for the automatic measurement of propofol. A mechanical switch controls the power-on of the board. At the power-on, some configurations and set-up operations are executed, to make the system ready to use. The configurations include the initialization of PWM and timer drivers, the definitions of interrupts and the BLE set-up. Once all the preliminary steps have been executed, the MCU starts BLE advertisement, and after the connection is established, it waits for the start command. During this state, the device acts as a Generic Attribute Profile (GATT) BLE server, which contains a set of GATT characteristics. The custom service is accessible by an external GATT client, which can call and read the measurement GATT characteristic. In this way, the device exits the idle-state to move to the on-state. The MCU drives the electrochemical cell with the $\mathrm{CV}$ pre-defined stimuli, modulating the PWM 
signal, and samples back the Faradaic current trough the QD signal. The sampling of QD is done by a simple counter which measures the timing distance between consecutive pulses of QD. The measurement is read directly by the client on the attribute "current" of the GATT characteristic.

\section{ML-Based Classifier}

An external computer, running custom software, and connected to a monitor, processes the measurement to provide smart and easy-to-read information to the anesthesiologist. This software contains a graphical user interface and a Support Vector Machine (SVM) classifier, taking advantages of [39], [40].

The ML-assisted method is required for compensating the fouling effect of propofol on the electrochemical sensor. As a sort of soft-modelling technique, instead of calibrating the sensor with a standard linear model, the SVM is previously trained on a set of know measure, and it learns from them the effect of the fouling phenomena on the measurement features. In this way, the algorithm is trained to compensate the non-linearities introduced by the degradation of the electrode. The classifier is the most efficient tool to directly provide to the final user (the anaesthesiologist) the primary information necessary to keep the constant-dose in the range of interest. On the computer resides the external GATT client, which reads via BLE the full vector of measurement samples. This data is stored and processed by the software to obtain a four-features list, which is sent to the ML-based model. Three relevant features are extracted from the received $\mathrm{CV}$, that are the peak current, the peak position, and the total charge, while the fourth feature is the ordinal number of measurements performed with the same sensor. A digital low-pass filter at the cut-off frequency of $2 \mathrm{~Hz}$ removes external electrical noise. The Faradaic current peak is detected after baseline subtraction. The peak is determined by the two features, which are the potential (position of the peak), and the current (height of the peak). These two features are the most relevant for characterizing the electrochemical reaction. Moreover, the total charge exchanged in the Faradaic process is extracted from the $\mathrm{CV}$ using the recently proposed Total Charge Detection in CV (TCDC) method [41].

In this work, kernelized-SVMs are implemented since SVMs are suitable for the classification of non-linear problems (such as fouling) building the optimal hyperplane in the space of the measurement features [40]. The SVM classifies the propofol concentration into six different categorical classes with values from zero to five representing concentration of $10 \mu \mathrm{M}, 20 \mu \mathrm{M}$, $30 \mu \mathrm{M}, 40 \mu \mathrm{M}, 50 \mu \mathrm{M}$, and $60 \mu \mathrm{M}$, respectively. Having previously performed the training on a set of known measurements, the SVM classifier is able to predict the unknown concentration of propofol present in a target human serum sample according to the similarity between the measurement features acquired and the trained instances.

As fully detailed in [40], the SVM has been developed over a Gaussian Radial Basis Function (RBF) kernel, and the training has been previously performed on a data-set of $600 \mathrm{CV}$ according to the target application. The processing and the algorithms are developed in Python 3.7.4, using NumPy and scikit-learn libraries [42]. The SVM classifier has been validated for the detection of propofol in undiluted human serum at the body temperature $\left(37{ }^{\circ} \mathrm{C}\right)$ to assess the performance. Namely, the SVM achieved a null-generalization error with $100 \%$ classification accuracy. With these results, the authors proved that the proposed system is capable of continuous monitoring of propofol for up to ten minutes, with one sample every half a minute, correctly predicting the concentration of propofol with classes of $10 \mu \mathrm{M}$ [40].

After ten minutes (twenty consecutive measure), the needleshaped sensor tip is saturated, and the ML algorithm is no more able to compensate non-linearities. For this reason, the sensor must be disposed of leaving space for a newer one. The jack connector helps to perform fast this replacement. After that, a button present on the GUI is pressed by the user to start the monitoring again. This operation sets to value zero the software parameter related to the ordinal number of measurements performed with the same sensor.

\section{MATERIALS AND METHODS}

Propofol (2,6-Diisopropylphenol) from Tokyo Chemical Industry Co., Ltd has been dissolved in $0.1 \mathrm{M}$ sodium hydroxide $(\mathrm{NaOH})$ the day of use to prepare a stock infusion of $5.4 \mathrm{mM}$ of propofol. Six different concentrations of propofol, equally spaced in its therapeutic range from $10 \mu \mathrm{M}$ and $60 \mu \mathrm{M}$ are synthetically prepared as samples for the analysis, dissolving the propofol stock solution in the background electrolyte in a backer in the lab environment.

The needle-shaped sensor is connected to Metrohm Autolab PGSTAT $302 \mathrm{~N}$ commercial lab potentiostat to perform its characterization. The samples are prepared in undiluted human serum, heat-inactivated from human male AB plasma, from Sigma-Aldrich;, right before use, and a hot plate stirrer from VWR; keeps the sample at $37^{\circ} \mathrm{C}$. The analysis of the samples is performed in $\mathrm{CV}$, at a Scan Rate (SR) of $0.1 \mathrm{~V} / \mathrm{s}$. The potentiostat drives the voltage from $-0.8 \mathrm{~V}$ up to $0.7 \mathrm{~V}$, with a potential step of $5 \mathrm{mV}, 30 \mathrm{~ms}$ long. The QD potentiostat is tested for the detection of propofol with PGE electrodes in Phosphate Buffer Saline (PBS) $10 \mathrm{mM}$ at $\mathrm{pH}$ 7.4. In this case, the $\mathrm{CV}$ is performed with an $\mathrm{SR}$ of $0.2 \mathrm{~V} / \mathrm{s}$, in the range between $-0.1 \mathrm{~V}$ and $1.1 \mathrm{~V}$, with a potential step of $6 \mathrm{mV}$, $30 \mathrm{~ms}$ long. The measurement has been repeated with the lab instrument (Metrohm Autolab PGSTAT $302 \mathrm{~N}$ ) to establish a fair comparison.

The calibration is evaluated in Matlab; (v. R2020a), with Signal Processing Toolbox ${ }^{\mathrm{TM}}$ (v. 8.4) and Curve Fitting Toolbox ${ }^{\mathrm{TM}}$ (v. 3.5.11). Findpeaks built-in function is used to detect the peaks in forms of local maxima, after a baseline (blank signal) rigid subtraction and digital filtering with lowpass function at $2 \mathrm{~Hz}$. The calibration curve is obtained by regression built-in function, which calculates the linear regression with a least-squares fit. The sensitivity of the sensor is the slope of the calibration curve. The Limit of Detection (LOD), which is the lowest concentration of drug detectable by the sensor, is computed as three times the standard deviation of the blank signal (in the absence of analyte) around the peak position, over the sensitivity. The linearity of 
interpolation is established via the coefficient of determination of linear regression $\left(\mathrm{r}^{2}\right)$.

\section{RESULTS}

All the components of the proposed system have been tested and validated to assess the performance of the complete system. The results cover the most challenging goals achieved by the proposed smart pen for the monitoring of anaesthetics, i.e. reproducibility, portability, and smartness. The needle-shaped sensor has been tested and characterized for propofol monitoring in human serum; finally, the QD potentiostat has been validated in comparison with respect to a commercial lab instrument, then the performance of the embedded device has been evaluated in terms of power consumption and portability.

\section{A. Detection of Propofol in Human Serum}

The needle-shaped sensor has been tested for the detection of propofol in undiluted human serum between $10 \mu \mathrm{M}$ and $60 \mu \mathrm{M}$. Previous works proved the strong relationship between temperature and calibration of electrochemical sensors [43]. For this reason, and given the target application, the calibration is performed at body temperature $\left(37^{\circ} \mathrm{C}\right)$. The performance of the sensor is evaluated through a seven-point calibration on three different items of the needle-shaped sensor. The results of this analysis are presented in Fig. 7. Fig. 7 a displays the output voltammogram (current upon voltage) obtained by a CV procedure on our sensor after data filtering and baseline subtraction. The Faradaic redox peak increases linearly with respect to the concentration of propofol present in the sample. The calibration curve (Fig. 7 b) displays the performance of the proposed sensor considering repeatability and reproducibility since it is obtained by an inter-electrode analysis. The relative standard deviation of the calibration point is $2.4 \%$ at $60 \mu \mathrm{M}$, with a maximum of $15.6 \%$ at $10 \mu \mathrm{M}$. The sensor sensitivity is $12.29 \pm 4.43 \mathrm{nA} / \mu \mathrm{M}$, with a relative standard deviation of $12.0 \%$. The linearity $\left(\mathrm{r}^{2}\right)$ is higher than $94.9 \%$. The LOD is $3.80 \pm 1.37 \mu \mathrm{M}$, below the minimum therapeutic concentration $(10 \mu \mathrm{M})$. The position of the redox peak is $452 \pm 110 \mathrm{mV}$. Considering the interference study, [24] discussed the interaction among the drugs in the cocktail of anaesthetics proving that it is possible to obtain simultaneous monitoring of these drugs (such as propofol and paracetamol).

\section{B. QD Potentiostat Validation}

The level of noise of the custom QD potentiostat has been estimated without the sensor, sampling the current with the QD signal every $10 \mathrm{~ms}$. The standard deviation of the current is $39 \mathrm{nA}$ resulting in 11.6 equivalent number of bits, considering the full range $( \pm 60 \mu \mathrm{A})$. The $\mathrm{QD}$ potentiostat is validated against the lab instrument (Metrohm Autolab PGSTAT 302 N). Fig. 8 displays the results of the comparison. The output voltammogram from the lab instrument (Fig. 8(a)) is compared directly to the output voltammogram from the proposed QD potentiostat (Fig. 8(b)). The different height and position of the peak are related to the linear offset introduced by the electronics, which does not change the measurement behaviour of the system since the curves do

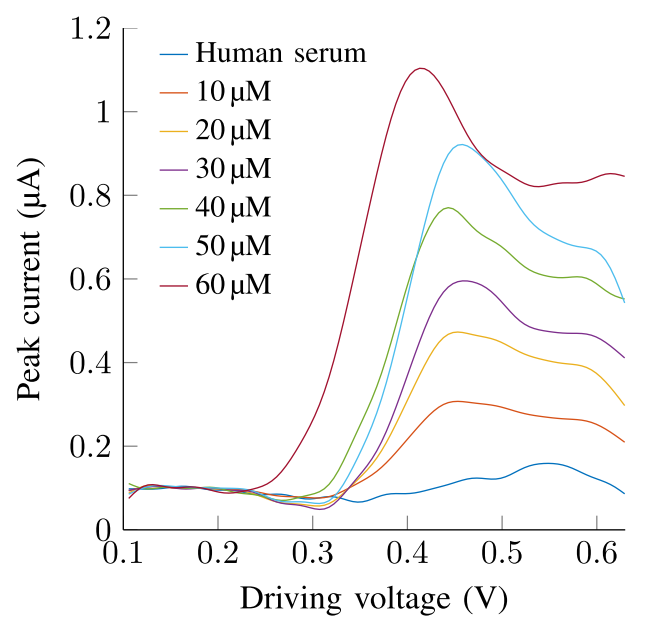

(a)

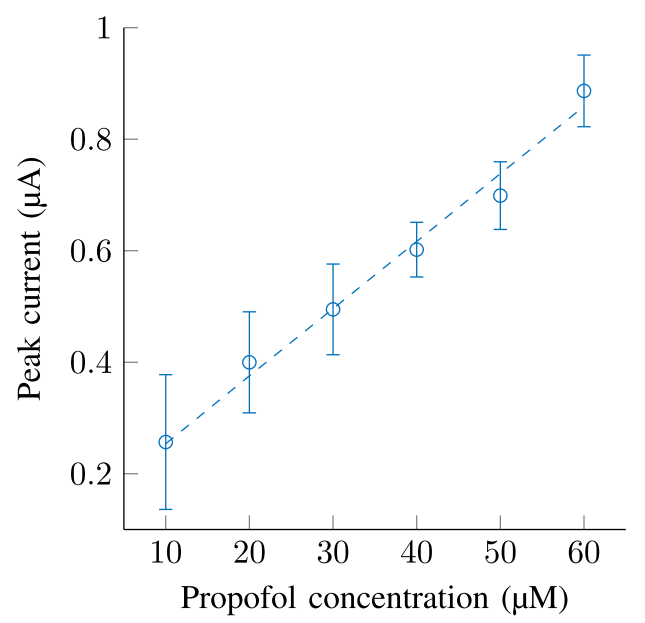

(b)

Fig. 7. Needle-shape sensor results: in the voltammogram (a) the current peak shows an increase which is linearly proportional to the concentration of propofol. This is used to extract the calibration curve (b).

not present any visible distortion. Fig. $8 \mathrm{c}$ reports the analysis of the data in the forms of calibration curves. The linearity $\left(\mathrm{r}^{2}\right)$ is higher than $99.9 \%$ in both cases, which demonstrates that the QD potentiostat does not introduce non-linearities. The sensitivity is $565 \mathrm{nA} / \mu \mathrm{M}$ with the lab instrument and $467 \mathrm{nA} / \mu \mathrm{M}$ with the proposed QD potentiostat.

\section{Power Consumption and Portability}

The power consumption of the board has been measured during lab testing, adding a digital multimeter in series on the power supply of the device. Table I reports the power consumption of the full embedded device both in idle-state and in on-state. The on-state considers a series of ten full voltammograms, acquired with a pause time of $30 \mathrm{~s}$, without connecting the sensor. The average absorbed current during the on-state is $20 \mathrm{~mA}$, corresponding to the average power consumption of $72 \mathrm{~mW}$. The QD potentiostat consumes $19.5 \mathrm{~mW}$ [30]. The digital control and the Bluetooth communication total average power is $52.5 \mathrm{~mW}$. 


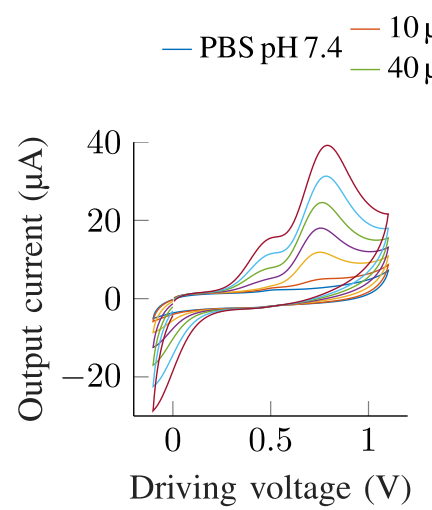

(a)

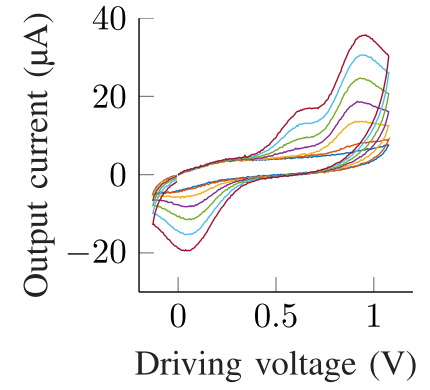

(b)

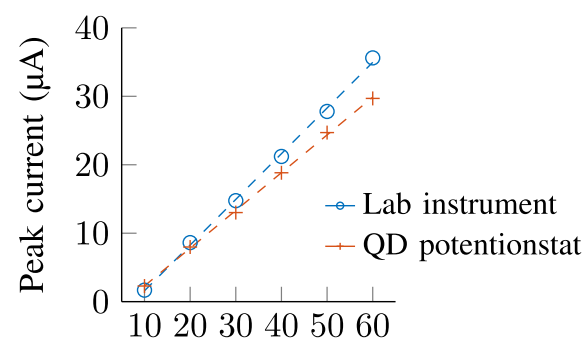

Propofol concentration $(\mu \mathrm{M})$

(c)

Fig. 8. Comparison between commercial lab instrument (a) and proposed QD potentiostat (b) in detection of propofol in its therapeutic range. The calibration curves (c) present few differences.

TABLE I

Total Power Consumption of THE EMBedded Device, Minimum (Min), Average (Avg), AND MaXimum (MaX) Values, Considering Both IDLE-STATE AND ON-STATE

\begin{tabular}{lccc}
\hline & Min & Avg & Max \\
\hline Total idle-state power $(\mathrm{mW})$ & 29.7 & 36.3 & 42.9 \\
Total on-state power $(\mathrm{mW})$ & 62.7 & 72.0 & 79.2 \\
\hline
\end{tabular}

TABLE II

COMPARISON With RESPECT TO THE STATE-OF-THE-ART

\begin{tabular}{lccccccc}
\hline & {$[18]$} & {$[21]$} & {$[22]$} & {$[24]$} & {$[25]$} & {$[26]$} & $\begin{array}{c}\text { This } \\
\text { work }\end{array}$ \\
\hline $\begin{array}{l}\text { Propofol LOD } \\
(\mu \mathrm{M})\end{array}$ & 0.5 & 0.1 & 0.7 & n.a. & n.a. & n.a. & 3.8 \\
$\begin{array}{l}\text { Number of } \\
\text { continuous } \\
\text { measure tested }\end{array}$ & 1 & 12 & 25 & 1 & n.a. & n.a. & 20 \\
$\begin{array}{l}\left.\text { Size (cm }{ }^{2}\right) \\
\begin{array}{l}\text { Power } \\
\text { consumption } \\
(\mathrm{mW})\end{array}\end{array}$ & n.a & n.a & n.a & 96 & 567 & n.a & 39 \\
\hline
\end{tabular}

The system features a 2.7 Ah battery, supporting a battery lifetime of 135 hours always-on, limiting the necessity of charges and allowing continuous usage in more than one surgery per charge. The BLE Received Signal Strength Indication (RSSI) has been estimated by the LightBlue; Android ${ }^{\mathrm{TM}}$ application to be $-95 \mathrm{dBm}$ at $18 \mathrm{~m}$ and $-51 \mathrm{dBm}$ at $0 \mathrm{~m}$, compliant with the BLE standard specifications.

\section{DISCUSSION}

To the extent of our knowledge, the literature presents just a few examples of anaesthetics monitoring systems including electronics and full devices with processing and display, such as using Raspberry Pi [25] or an IoT Cloud [26]. Table I presents a full comparison with respect to the state-of-the-art ([18], [21], [22], [24]-[26]). The sensor features a LOD higher with respect to the state-of-the-art but remains below the minimum concentration target of our reference application $(10 \mu \mathrm{M})$. This work, thanks to the results obtained in [40], has been tested for continuous monitoring up to twenty consecutive measurements with the same sensor, similarly to what achieved by the literature [21], [22]. Our device is 59\% smaller (w.r.t. [24]) and consumes way less than one fifth of the total power (w.r.t. [26]). Moreover, to the best of our knowledge, the proposed system is the only one presenting a fully-wireless device and including ML-based smart processing. The latter one compensates non-linearities and displays with $100 \%$ classification accuracy the results [40].

\section{CONCLUSION}

We presented a novel smart pen for continuous monitoring of propofol anaesthetic drug. The presented electronic device is the missing piece of technology to close the loop between anaesthesiologist and patient with TDM towards safer anaesthesiology practices. The system is developed upon a single wireless battery-operated embedded device in a pen-shaped case, 59\% smaller and $81 \%$ less power consuming w.r.t. the state-of-the-art, allowing full portability and enhancing mobility thanks to QD approach. The sensor LOD $(3.80 \pm 1.37 \mu \mathrm{M})$ fits the requirements of the medical application, directly in undiluted human serum at $37^{\circ} \mathrm{C}$, with $100 \%$ accurate ML-based classifier. Future works will include full assembly of the system, development of a microcatheter for the blood sampling, and the start of the clinical test.

\section{ACKNOWLEDGMENT}

The authors would like to thank Dr. Francesca Criscuolo for her helpful advice and Dr. Alessandro Sanginario for his help in 3D printing.

\section{REFERENCES}

[1] R. D. Miller, L. Eriksson, L. A. Fleisher, J. P. Wiener-Kronish, and W. L. Young, "Miller's anesthesia," in Miller's Anesthesia.: Amsterdam, Netherlands: Elsevier, 2010, pp. 2827-2827.

[2] M. J. Murray et al., Faust's Anesthesiology Review E-Book: Expert Consult. Amsterdam, The Netherlands: Elsevier Health Sciences, 2014.

[3] S. A. K. Craig and R. Kitson, "Risks associated with anaesthesia," Anaesth. Intensive Care Med., vol. 11, no. 11, pp. 464-468, 2010.

[4] Z. Al-Rifai and D. Mulvey, "Principles of total intravenous anaesthesia: Basic pharmacokinetics and model descriptions," Bja Educ., vol. 16, no. 3, pp. 92-97, 2016.

[5] Y. Park, S. H. Han, W. Byun, J. H. Kim, H. C. Lee, and S. J. Kim, “A real-time depth of anesthesia monitoring system based on deep neural network with large EDO tolerant EEG analog front-end," IEEE Trans. Biomed. Circuits Syst., vol. 14, no. 4, pp. 825-837, Aug. 2020. 
[6] R. Eyres, "Update on TIVA," Pediatr. Anesth., vol. 14, no. 5, pp. 374-379, 2004.

[7] Y. Wahlquist, K. van Heusden, G. A. Dumont, and K. Soltesz, "Individualized closed-loop anesthesia through patient model partitioning," in Proc. IEEE 42nd Annu. Int. Conf. Eng. Med. Biol. Soc., 2020, pp. 361-364.

[8] K. Patlatzoglou, S. Chennu, O. Gosseries, V. Bonhomme, A. Wolff, and S. Laureys, "Generalized prediction of unconsciousness during propofol anesthesia using 3D convolutional neural networks," in Proc. 42nd IEEE Annu. Int. Conf. Eng. Med. Biol. Soc., Montreal, Canada, 2020, pp. 134137.

[9] G. Wang et al., "Monitoring the depth of anesthesia through the use of cerebral hemodynamic measurements based on sample entropy algorithm," IEEE Trans. Biomed. Eng., vol. 67, no. 3, pp. 807-816, Mar. 2020.

[10] K. van Heusden, M. Yousefi, J. M. Ansermino, and G. A. Dumont, "Closedloop MISO identification of propofol effect on blood pressure and depth of hypnosis," IEEE Trans. Control Syst. Technol., vol. 28, no. 1, pp. 254-263, Jan. 2020.

[11] M. Jeanne, B. Tavernier, R. Logier, and J. De Jonckheere, "Closed-loop administration of general anaesthesia: From sensor to medical device," Pharmaceut. Technol. Hosp. Pharm., vol. 2, no. 2, pp. 63-70, 2017.

[12] T. Buclin et al., "The steps to therapeutic drug monitoring: A structured approach illustrated with imatinib," Front. Pharmacol., vol. 11, p. 177, 2020.

[13] J. Kanto and E. Gepts, "Pharmacokinetic implications for the clinical use of propofol," Clin. Pharmacokinetics, vol. 17, no. 5, pp. 308-326, 1989.

[14] A. Simalatsar, M. Guidi, P. Roduit, and T. Buclin, "Robustness analysis of personalised delivery rate computation for IV administered anesthetic," Smart Health, vol. 9, pp. 101-114, 2018.

[15] M. Grossherr, A. Hengstenberg, T. Meier, L. Dibbelt, K. Gerlach, and H. Gehring, "Discontinuous monitoring of propofol concentrations in expired alveolar gas and in arterial and venous plasma during artificial ventilation," J. Amer. Soc. Anesthesiol., vol. 104, no. 4, pp. 786-790, 2006.

[16] F. Zhang et al., "A non-invasive monitoring of propofol concentration in blood by a virtual surface acoustic wave sensor array," Anal. Sci., vol. 33, no. 11, pp. 1271-1277, 2017.

[17] H. Dong et al., "Evaluating propofol concentration in blood from exhaled gas using a breathing-related partition coefficient," Anesth. Analg., vol. 130, no. 4, pp. 958-966, 2020.

[18] C.-C. Hong, C.-C. Lin, C.-L. Hong, Z.-X. Lin, M.-H. Chung, and P.-W. Hsieh, "Handheld analyzer with on-chip molecularly-imprinted biosensors for electrical detection of propofol in plasma samples," Biosensors Bioelectron., vol. 86, pp. 623-629, 2016.

[19] C. Dincer et al., "Disposable sensors in diagnostics, food, and environmental monitoring," Adv. Mater, vol. 31, no. 30, 2019, Art. no. 1806739.

[20] X. Yang, J. Kirsch, J. Fergus, and A. Simonian, "Modeling analysis of electrode fouling during electrolysis of phenolic compounds," Electrochimica Acta, vol. 94, pp. 259-268, 2013.

[21] F. Rainey, F. Kivlehan, E. Chaum, and E. Lindner, "Toward feedback controlled anesthesia: Automated flow analytical system for electrochemical monitoring of propofol in serum solutions," Electroanalysis, vol. 26, no. 6, pp. 1295-1303, 2014

[22] F. Stradolini, T. Kilic, A. Di Consiglio, M. Ozsoz, G. De Micheli, and S. Carrara, "Long-term monitoring of propofol and fouling effect on pencil graphite electrodes," Electroanalysis, vol. 30, no. 7, pp. 1363-1369, 2018.

[23] A. Tuoheti et al., "New approach for making standard the development of biosensing devices by a modular multi-purpose design," IEEE Trans. Nanobiosci., vol. 19, no. 3, pp. 339-346, Jul. 2020.

[24] F. Stradolini, T. Elboshra, A. Biscontini, G. De Micheli, and S. Carrara, "Simultaneous monitoring of anesthetics and therapeutic compounds with a portable multichannel potentiostat," in Proc. IEEE Int. Symp. Circuits Syst., 2016, pp. 834-837.

[25] F. Stradolini, A. Tuoheti, P. Motto Ros, D. Demarchi, and S. Carrara, "Raspberry pi based system for portable and simultaneous monitoring of anesthetics and therapeutic compounds," in Proc. New Gener. CAS (NGCAS), 2017, pp. 101-104.

[26] F. Stradolini et al., "An IoT solution for online monitoring of anesthetics in human serum based on an integrated fluidic bioelectronic system," IEEE Trans. Biomed. Circuits Syst., vol. 12, no. 5, pp. 1056-1064, Oct. 2018.

[27] A. Ainla et al., "Open-source potentiostat for wireless electrochemical detection with smartphones," Anal. Chem., vol. 90, no. 10, pp. 6240-6246, 2018.

[28] S. Aiassa, S. Carrara, and D. Demarchi, "Optimized sampling rate for voltammetry-based electrochemical sensing in wearable and IoT applications," IEEE Sens. Lett., vol. 3, no. 6, pp. 1-4, Jun. 2019.
[29] S. Aiassa, S. Yilmaz, S. Carrara, and D. Demarchi, "Pencil graphite needleshaped biosensor for anaesthetic monitoring in human serum," in Proc. IEEE Sensors, Rotterdam, Netherlands, 2020, pp. 1-4.

[30] S. Aiassa, F. Stradolini, A. Tuoheti, S. Carrara, and D. Demarchi, "Quasidigital biosensor-interface for a portable pen to monitor anaesthetics delivery," in Proc. 15th Conf. Ph.D Res. Microelectronics Electron. (PRIME), Lausanne, Switzerland, 2019, pp. 265-268.

[31] S. Aiassa, P. Motto Ros, G. Masera, and M. Martina, "A low power architecture for AER event-processing microcontroller," in Proc. IEEE Biomed. Circuits Syst. Conf., 2017, pp. 1-4.

[32] P. Motto Ros, A. Sanginario, M. Crepaldi, and D. Demarchi, "Qualityenergy trade-off and bio-inspired electronic systems," in Proc. IEEE Int. Conf. Sci. Elect. Eng. Isr., 2018, pp. 1-5.

[33] P. Motto Ros, M. Crepaldi, A. Damilano, and D. Demarchi, "Integrated bio-inspired systems: An event-driven design framework," in Proc. 10th Int. Conf. Innov. Inf. Technol., Abu Dhabi, United Arab Emirates, 2014, pp. $48-53$.

[34] A. Damilano et al., "A robust capacitive digital read-out circuit for a scalable tactile skin," IEEE Sensors J., vol. 17, no. 9, pp. 2682-2695, May 2017

[35] O. L. Nuzumlali, "Detailed noise analysis of current-to-frequency converters for precision analog accelerometers," in Proc. IEEE/ION Position Location Navigation Symp., Savannah, Georgia, USA, 2016, pp. 898-904.

[36] M. Crepaldi, M. Stoppa, P. Motto Ros, and D. Demarchi, "An analogmode impulse radio system for ultra-low power short-range audio streaming," IEEE Trans. Circuits Syst. I: Regular Papers, vol. 62, no. 12, pp. 2886-2897, Dec. 2015.

[37] F. Rossi, P. Motto Ros, R. M. Rosales, and D. Demarchi, "Embedded biomimetic system for functional electrical stimulation controlled by eventdriven sEMG," Sensors, vol. 20, no. 5, p. 1535, 2020.

[38] D. Tunzi, "Portable embedded system for continuous monitoring of anaesthetics," Master's thesis, Politecnico di Torino, Turin, Italy, 2019.

[39] S. Aiassa, F. Grassi, R. Terracciano, S. Carrara, and D. Demarchi, "Live demonstration: Quasi-digital portable pen to monitor anaesthetics delivery," in Proc. IEEE Biomed. Circuits Syst. Conf., 2019, p. 1.

[40] S. Aiassa et al., "Continuous monitoring of propofol in human serum with fouling compensation by support vector classifier," Biosensors Bioelectron., vol. 171, 2021, Art. no. 112666.

[41] S. Aiassa, J. D. Martínez González, D. Demarchi, and S. Carrara, "New measurement method in drug sensing by direct total-charge detection in voltammetry," in Proc. IEEE Int. Symp. Med. Meas. Appl., Bari, Italy, 2020, pp. 1-6.

[42] F. Pedregosa et al., "Scikit-learn: Machine learning in python," J. Mach. Learn. Res., vol. 12, no. 2011, pp. 2825-2830, 2011.

[43] S. L. Ntella, F. Stradolini, A. Tuoheti, D. Demarchi, A. A. Hatzopoulos, and S. Carrara, "Architecture and procedures for $\mathrm{pH}$ and temperature monitoring in medical applications," in Proc. IEEE SENSORS, Glasgow, U.K., 2017, pp. 1-3.

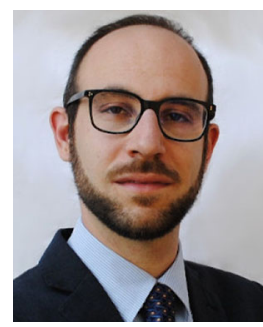

Simone Aiassa (Student Member, IEEE) received the B.S. and M.S. degrees in electronic engineering with Politecnico di Torino, Turin, Italy, in 2014 and 2017, respectively. Since 2017, he has been working toward the Ph.D. degree in electronic engineering with the Department of Electronics and Telecommunications, Politecnico di Torino and a Member of Micro\&Nano Electronic Systems Group, developing embedded systems for biomedical applications. From 2018 to 2020, he was a Guest Ph.D. Student with the Integrated Systems Laboratory, École Polytechnique Fédérale de Lausanne, implementing a system for continuous monitoring of anaesthetics. In 2007, he was M.S. Thesis Student with Istituto Italiano di Tecnologia. He is the author of 15 academic papers and a Reviewer in more than 50 publications. His research interests include digital-design, bio-inspired electronics, electrochemical sensing, and biomedical circuit and systems. $\mathrm{He}$ was the recipient of the Best Paper Award at IEEE MeMeA 2020 and the 2019 Ph.D. Quality Award at Politecnico di Torino. In 2020, he was the Chair of the Politecnico di Torino IEEE Student Branch awarded as Exemplary Student Branch in Italy Section and Region 8. 


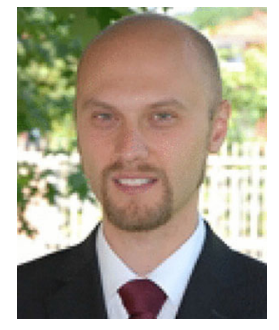

Paolo Motto Ros (Member, IEEE) received the Engineering and $\mathrm{Ph} . \mathrm{D}$. degrees in electronic engineering from the Politecnico di Torino (IT), Turin, Italy, in 2005 and 2009, respectively. He is a Senior Postdoctoral Researcher and an Adjunct Professor with Micro\&Nano Electronic Systems Group, Dipartimento di Elettronica e Telecomunicazioni, Politecnico di Torino (IT). From 2009 to 2012, he was with Politecnico di Torino as a Postdoc Researcher jointly with, 2009-2013, Istituto Nazionale Fisica Nucleare, INFN, IT. From 2012 to 2019, he was with Istituto Italiano di Tecnologia, where since 2014, he has been a Senior Postdoc Researcher. He authored or coauthored more than 60 publications. His current research interests include event-driven digital integrated circuits, architectures, and systems, low-power smart sensor networks, bio-inspired electronics, biomedical, and humanoid robotic applications. He is a Member of the IEEE CAS Society. He was a Member of the Organizing Staff of the IEEE BioCAS 2017 conference, and a Member of the Organizing Committee of the IEEE ICECS 2019 conference and the FoodCAS Satellite Event at the IEEE ISCAS 2021 conference. He is the Guest Editor of the MDPI Sensors and the Topic Editor of the Frontiers in Neurorobotics.

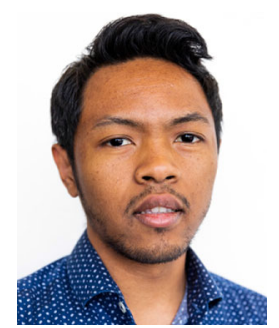

Mandresy Ivan Ny Hanitra (Student Member, IEEE) received the B.S. degree in physics and chemistry from the Grenoble Institute of Technology, Grenoble, France, in 2014, the M.S. degree in micro and nanotechnologies for integrated systems and a joint degrees from the Politecnico di Torino, Turin, Italy, the Grenoble Institute of Technology, and EPFL, Lausanne, Switzerland, in 2016, and the master's degree from the Electronics Laboratory (Prof. Kayal), EPFL, developing low-power systems for photoplethysmographic-based heart rate monitoring. $\mathrm{He}$ is currently working toward the Ph.D. degree in microelectronics and microsystems. In October 2016, he joined the Integrated Systems Laboratory (Prof. De Micheli), EPFL, where he designed and realized multitarget electrochemical sensing platforms. His research interests include the co-design and development of electronic interfaces for electrochemical sensors. A strong emphasis of his research is laid on developing efficient data processing tools and optimization algorithms within a multisensing paradigm. His work is aiming at physical status monitoring and advanced healthcare diagnosis for wearable applications.

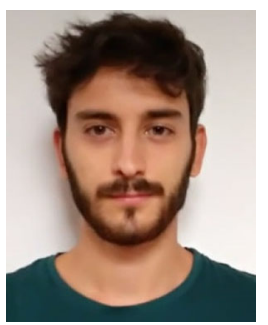

Davide Tunzi received the B.S. degree in electronic and telecommunication engineering from Politecnico di Bari, Bari, Italy and the M.S. degree in electronic engineering at Politecnico di Torino, Turin, Italy, in 2017 and 2019, respectively. He has developed his thesis project in the design of the embedded electronic systems for continuous monitoring of anaesthetics in 2019. He currently works in the area of the embedded electronic system in particular in the design of metering and charging device and system.

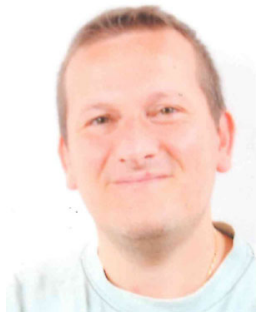

Maurizio Martina (Senior Member, IEEE) received the M.S. and Ph.D. degrees in electrical engineering from the Politecnico di Torino, Turin, Italy, in 2000 and 2004, respectively. He is currently an Associate Professor with the VLSI-Lab Group, Politecnico di Torino. He edited one book and authored or coauthored three book chapters on VLSI architectures and digital circuits for video coding, wireless communications, and error correcting codes. He has more than 100 scientific publications and is the author of two patents. His research focuses on VLSI design and implementation of architectures for digital signal processing, video coding, communications, artificial intelligence, machine learning, and event-based processing. He is currently an Associate Editor for the IEEE TRANSACTIONS ON CIRCUITS AND SYSTEMS-I: REGULAR PAPERS. He was a part of the Organizing and a Technical Committee of several international conferences, including BioCAS 2017, ICECS 2019, and AICAS 2020. He is also the Counselor of the IEEE Student Branch at the Politecnico di Torino and a Professional Member of IEEE HKN.

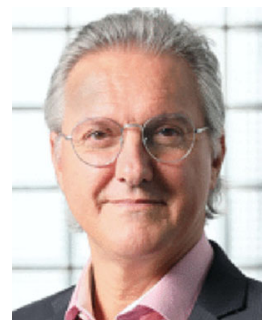

Sandro Carrara (Fellow, IEEE) received the Ph.D. degree in biochemistry and biophysics from the University of Padua, Padua, Italy, the master's degree in physics from the University of Genoa, Genoa, Italy, and a Diploma in electronics from the National Institute of Technology, Albenga, Italy. He is currently a Faculty with the EPFL in Lausanne $(\mathrm{CH})$, a former Professor with the Universities of Genoa and Bologna. He has authored or coauthored seven books with prestigious publishers, which include Springer Nature and Cambridge University Press. He has more than 300 scientific publications and is the author of 14 patents. He is Editor-inChief of the IEEE SENSORS JOURNAL, one of the largest journals among 200 IEEE publications, and an Associate Editor for the IEEE TRANSACTIONS ON Biomedical Circuits AND Systems. He is a Member of the IEEE Sensors Council and his Executive Committee. He was a Member of the Board of Governors of the IEEE Circuits And Systems Society. He was the recipient of the IEEE Sensors Council Technical Achievement Award.

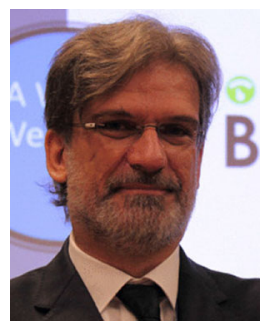

Danilo Demarchi (Senior Member, IEEE) received the Engineering and $\mathrm{Ph} . \mathrm{D}$. degrees in electronics engineering from Politecnico di Torino, Torino, Italy, in 1991 and 1995, respectively. He is currently an Associate Professor with the Department of Electronics and Telecommunications, Politecnico di Torino. He is or was a Visiting Professor with EPFL Lausanne, Lausanne, Switzerland and with Tel Aviv University, Tel Aviv, Israel. In 2018, he was Visiting Scientist with the Massachusetts Institute of Technology and Harvard Medical School, Cambridge, MA, USA. He has authored and coauthored five patents and more than 250 scientific publications in international journals and peer-reviewed conference proceedings. He is leading the Micro\&Nano Electronic Systems Laboratory of Politecnico di Torino. He was a Member of the BioCAS Technical Committee, an Associate Editor for the IEEE TRANSACTIONS ON BIOMEDICAL CIRCUITS AND SySTEMS, IEEE SENSORS, and of the Springer Journal BioNanoScience, the General Chair of Biomedical Circuits and Systems Conference edition in Torino, October 2017, and the Founder of IEEE FoodCAS Workshop (Circuits and Systems for the Food Chain). 